\title{
Hiding greenhouse gas emissions in the cloud
}

To the Editor - Data centres account for $200 \mathrm{TWh} \mathrm{yr}^{-1}$, or around $1 \%$ of total global electricity demand ${ }^{1}$. While their energy usage has been stable in recent years as efficiencies increase, it may grow to between $15-30 \%$ of electricity consumption in some countries by 2030 (ref. $^{2}$ ).

The majority of this growth is attributed to cloud computing, particularly by the largest 'hyperscale' vendors such as Amazon, Google and Microsoft. The market size for cloud computing has grown from $\$ 6$ billion in 2008 to $\$ 288$ billion in 2019 (ref. ${ }^{3}$ ), with the majority of future growth in information technology (IT) workloads across all types of data centres expected to be in the cloud ${ }^{4}$.

If outsourced to the cloud, IT emissions previously accounted for under the Greenhouse Gas Protocol ${ }^{5}$ Scope 1 emissions that are directly linked to the activities of an organization from sources that it owns and control - and Scope 2 - emissions from the generation of purchased energy - move to Scope 3, referring to all other emissions. When an organization purchases its own equipment and signs contracts for electricity from a co-location facility, all their IT emissions fall under Scope 1 or Scope 2 (ref. ${ }^{6}$ ). If the IT workload is moved to the cloud, the organization is no longer directly generating emissions under Scope 1 and is not purchasing energy under Scope 2. Therefore, all those emissions move to Scope 3.

Currently, Scope 3 emissions tend to be reported only voluntarily, and the emissions are aggregated into the global emissions reporting by the large cloud vendors ${ }^{7-9}$, which can result in individual corporation's emissions being hidden when they are moved to the cloud. This is an issue, as where national reporting rules require disclosure of emissions statistics, only Scope 1 and Scope 2 emissions are compulsory (for example, see ref. ${ }^{10}$ ).

Deploying in the cloud is claimed to be significantly greener than the old data centre approach, as the technology sector is the largest purchaser of renewables ${ }^{2}$ and the major cloud providers compete to be the greenest. Google is carbon neutral and has purchased renewable electricity to match $100 \%$ of its electricity usage since 2017 (ref. ${ }^{8}$ ); Microsoft, also carbon neutral, has pledged to match renewables by 2025 (ref. ${ }^{11}$ ), as has Amazon ${ }^{12}$.

The problem lies in the lack of transparency. None of the data required to calculate the greenhouse gas (GHG)

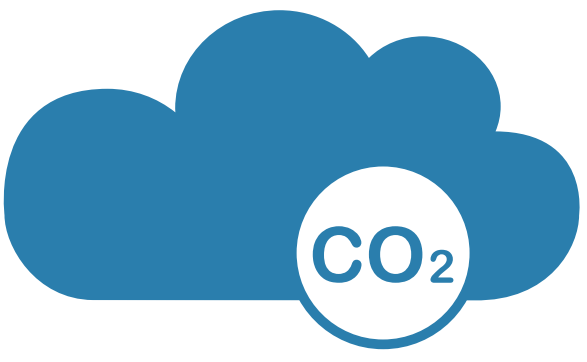

Credit: KRISSIKUNTERBUNT / ALAMY STOCK VECTOR

emissions of an organization is available once they move to the cloud. Cloud vendors' aggregated global reports do not provide the data needed to understand the environmental footprint. If an organization is implementing reporting under International Organization for Standardization (ISO) 14064-1 then it will be unable to attribute its 'other indirect emissions', the ISO standard category equivalent of Scope 3 (ref. ${ }^{13}$ ). Further, if an organization wishes to mitigate its emissions, such as through offsets, then the only option is to rely on the cloud vendor to do it for them.

A 2019 report shows how challenging it is to make estimates without data and highlights the lack of transparency at a local level ${ }^{14}$. The report methodology attempted to estimate data centre sizes from Freedom of Information requests combined with data from lease and energy contracts. Even this only allows for estimates of energy usage in a particular region - more granular data from inside the data centre is needed to understand and accurately account for emissions from cloud workloads.

The good news is that this may be changing. At the beginning of 2020, Microsoft announced the availability of their Sustainability Calculator ${ }^{15}$. This is a tool for their Azure cloud customers, which provides a GHG Protocol compliant report of the emissions from purchased cloud resources. However, it is currently limited to 'Enterprise' customers and is an add-on to a commercial reporting product, PowerBI, which means it is not generally available to every Azure customer. Despite this, this tool may be the catalyst needed to encourage others to do the same. Even if the underlying data to make the calculations is not available, the final emissions calculation is the most important figure.
As more workloads move to the cloud, more companies wish to demonstrate their environmental credentials, and as mandatory reporting is adopted by more countries, it is important that GHG emissions figures continue to be calculated. Transparent and consistent financial accounting rules allow for companies to be analysed and compared - the same should be expected for environmental reporting.

\section{David Mytton (D)}

Centre for Environmental Policy, Imperial College

London, London, UK.

$\bigotimes_{\text {e-mail: david@davidmytton.co.uk }}$

Published online: 13 July 2020

https://doi.org/10.1038/s41558-020-0837-6

References

1. Jones, N. Nature 561, 163-167 (2018).

2. Kamiya, G. \& Kvarnström, O. Data centres and energy - from global headlines to local headaches? International Energy Agency https://www.iea.org/commentaries/data-centres-and-energyfrom-global-headlines-to-local-headaches (2019).

3. Adams, J. \& Cser, A. Forrester Data: Cloud Security Solutions Forecast, 2016 To 2021 (Global) (Tata Communications, 2019); https://www.tatacommunications.com/wp-content/ uploads/2019/02/Forrester-Report.pdf

4. Shehabi, A. et al. United States Data Center Energy Usage Report Report No. LBNL-1005775 (Lawrence Berkeley National Laboratory, 2016); https://eta.lbl.gov/publications/united-statesdata-center-energy

5. The Greenhouse Gas Protocol: A Corporate Accounting and Reporting Standard (World Business Council for Sustainable Development and World Resources Institute, 2015); https://ghgprotocol.org/corporate-standard

6. The Greenhouse Gas Protocol: ICT Sector Guidance built on the GHG Protocol Product Life Cycle Accounting and Reporting Standard (Global eSustainability Initiative, 2017); https://ghgprotocol.org/ sites/default/files/GHGP-ICTSG\%20-\%20ALL\%20Chapters.pdf

7. Carbon footprint. Amazon https://sustainability.aboutamazon. com/carbon-footprint (2020).

8. Google Environmental Report 2019 (Google, 2019); https:// services.google.com/fh/files/misc/google_2019-environmentalreport.pdf

9. 2018 Data Factsheet: Environmental Indicators (Microsoft, 2018); http://query.prod.cms.rt.microsoft.com/cms/api/am/binary/ RE3455q

10. Consultation Outcome: Streamlined Energy and Carbon Reporting (UK Government, 2018); https://www.gov.uk/government/ consultations/streamlined-energy-and-carbon-reporting

11. Smith, B. Microsoft will be carbon negative by 2030 . Official Microsoft Blog https://blogs.microsoft.com/blog/2020/01/16/ microsoft-will-be-carbon-negative-by-2030/ (2020).

12. Amazon announces $\$ 2$ billion climate pledge fund to invest in companies building products, services, and technologies to decarbonize the economy and protect the planet. Amazon https://press.aboutamazon.com/news-releases/news-releasedetails/amazon-announces-2-billion-climate-pledge-fund-investcompanies (2020).

13. Greenhouse Gases - Part 1: Specification with Guidance at the Organization Level for Quantification and Reporting of Greenhouse Gas Emissions and Removals (ISO, 2018); https://www.iso.org/ standard/66453.html

14. Cook, G., Jardim, E. \& Craighill, C. (eds) Clicking Clean Virginia: The Dirty Energy Powering Data Center Alley (Greenpeace, 2019); https://www.greenpeace.org/usa/reports/click-clean-virginia/

15. Microsoft sustainability calculator. Microsoft https://appsource. microsoft.com/en-us/product/power-bi/coi-sustainability. sustainability_dashboard (2020). 